\title{
Structural Requirements for Digital Transformation - Insights from German Enterprises
}

\author{
Matthias Murawski ${ }^{1}$, Tristan Thordsen ${ }^{1}$, Malte Martensen ${ }^{2}$, Christina Rademacher ${ }^{3}$ \\ and Markus Bick ${ }^{1}$ \\ ${ }^{1}$ ESCP Europe Business School Berlin, Berlin, Germany \\ $\{$ mmurawski, tthordsen, mbick\} descpeurope.eu \\ ${ }^{2}$ IUBH University of Applied Sciences, Berlin, Germany \\ m.martensen@iubh. de \\ ${ }^{3}$ Promerit AG, Munich, Germany \\ christina.rademacherepromerit.com
}

\begin{abstract}
German enterprises are often characterised by low levels of digital maturity. One reason for this is a lack of required structural changes on the path towards digital transformation. We consider a prescriptive framework, the digital transformation framework (DTF), which contains four structural requirements for digital transformation. Based on 16 interviews with German digitalisation experts, we aim at an evaluation of the DTF. The outcome is an enriched version of it containing seven structural requirements including the newly identified factors culture of change, agility of organizational structure, and integration of cloud computing and platforms. The extended DTF sheds light on additional facets of the digital transformation and thus supports managers in navigating their undertaking in this dynamic environment. Corresponding implications for research and practice are discussed.
\end{abstract}

Keywords: Digital Transformation, Evaluation, Interviews, Prescriptive Framework, Structural Requirements

\section{Introduction}

Digital transformation is characterised by converging social, mobile, analytics, and cloud (SMAC) technologies and continuing miniaturisation with increasing processing power, storage capacity, and communication bandwidth [1]. Digital transformation denotes the changes digital technologies can cause with regards to a company's business model, resulting in adapted products or organizational structures [2]. Consequently, digital technologies have the power to complement and enrich existing products and services and enable entirely new business models [3]. In the context of this enormous and often radical change of the business landscape, new ways to cope with the situation and to develop strategic approaches are required by managers [4]. However, many companies struggle with their digital transformation. This is especial- 
ly true for the German business landscape, which shows a relatively low level of digital transformation compared to other countries [e.g. 5].

But what are the reasons for this lack of digital maturity of German business? In this paper we focus on one potential aspect: structural requirements. Structural requirements refer to the organisational setup of a company and refer to "organizational structures, processes and skill sets that are necessary to cope with and exploit new technologies" [2, p. 124]. Thus, the focus lies on the integration of digital technology into the organisational structure [6]. Related examples are new roles and responsibilities such as the Chief Digital Officer [7], or the recruitment of employees who possess and maintain high levels of digital competency [8]. Structural requirements are deemed necessary for an organisation's digital transformation [2]. Companies that fail to consider these aspects face a higher risk of failing in their efforts toward digital transformation [9].

However, there is little empirical research regarding specific structural requirements for digital transformation [10]; especially lacking are comprehensive overviews of these factors. For instance, Holotiuk and Beimborn [11] analysed 21 industry reports and identified organisational requirements and structural changes as critical success factors for a digital transformation, but also found that these factors require further in-depth analyses.

Structural requirements for digital transformation are primarily mentioned in studies dealing with the development of digital strategies [12-14]. On a more concrete level, Hess et al. [2] suggest four structural topics required in their digital transformation framework (DTF): operational change, responsibility for digital transformation, integration of new activities, and building of organisational competencies. Compared to most existing papers on digital transformation, the DTF is a guideline with a significant practical value, as it offers so-called strategic questions for managers regarding digital transformation. ${ }^{1}$ Due to its outstanding practical value and the pioneer character of the DTF, we have chosen this framework as model of reference. The DTF can be considered as prescriptive since it supports managers in their decision making in the context of digital transformation. This is in line with recent calls for more relevance and practical value of IS research [15]. However, it must be noted that the empirical basis of the DTF are three case studies from the media industry. Although Hess et al. formulate their framework in a way that it is applicable to other industries as well, we believe that confronting the DTF with findings from other domains, and consequently a potential update of the DTF, would strengthen its applicability. Thus, the objective of this study is to assess and, if required, to extend the DTF by naturalistic evaluation, meaning that findings from other domain's experts are analysed and integrated. Therefore, while placing the focus on German organisations, we formulate three research questions:

1 This statement can be underscored by the fact that we have used the DTF of Hess et al. for developing digital strategies during executive education programmes at our business school. Overall feedback of the executives on the DTF was positive, especially regarding its 'practical value'. 
RQ1: Which structural requirements identified in our cross-domain analysis are not part of the initial DTF of Hess et al. (2016)?

$R Q 2:$ Which structural requirements identified in our cross-domain analysis are already part of the initial DTF of Hess et al. (2016)?

RQ3: Which structural requirements of the initial DTF of Hess et al. (2016) could not be identified in our cross-domain analysis?

The paper is structured as follows. First, we present the theoretical basis - the digital transformation framework (DTF) of Hess et al. [2] - in the theoretical background section. In the research design section, we outline our research approach before results are described in the findings section. In the discussion, we debate our findings; our paper ends with a conclusion.

\section{Theoretical background}

The role of technologies and their impact on firms has led to a substantial rethinking of the strategic role of IT among scholars and practitioners alike. In recent decades the so-called alignment view [16], which postulates IT strategy as aligned with but also subordinate to business strategy, has been the dominant perspective. Obviously, due to the transforming power of digital technologies $[17,18]$ and the corresponding increased importance of IT strategy, the alignment view no longer reflects the actual situation. Thus, IS scholars have argued for a fusion between IT strategy and business strategy, which is called digital business strategy (DBS) and defined as "organisational strategy formulated and executed by leveraging digital resources to create differential value" $[19$, p. 472].

Although DBS has attracted remarkable attention (e.g., a special issue of MIS Quarterly in 2013), its lack of transformational aspects has been criticised [2, 6]. Matt et al., for example, have proposed an enhancement of DBS, focusing on the digital transformation strategy (DTS). In contrast to DBS, which emphasises describing desired future opportunities or strategies, DTS "is a blueprint that supports companies in governing the transformations that arise owing to the integration of digital technologies, as well as in their operations after the transformation" [6, p. 340].

Furthermore, Matt et al. [6] transfer their DTS into a digital transformation framework (DTF). Given this conceptual basis, Hess et al. [2] suggest a DTF with concrete strategic questions - a prescriptive model - which can be used to develop a digital transformation strategy. The DTF consists of the four dimensions: use of technologies, changes in value creation, structural changes and financial aspects. Hess et al. [2] develop a set of corresponding strategic questions relevant for digital transformation. Moreover, they provide potential answer categories for each of the questions but emphasise that "there are no universal, definitive answers" to them [2, p. 137].

Given the research goal of our study, to explore structural requirements for digital transformation and to evaluate the DTF of Hess et al. [2], we place our focus on structural changes. This dimension refers to "variations in a firm's organisational setup, especially concerning the placement of the new digital activities within the corporate 
structures" [6, p. 341] which equals our understanding of structural requirements in our study. Hess et al. [2] suggest four structural requirements: responsibility for digital transformation, organisational positioning of new activities, operational changes, and building of competencies. In the following sections of this paper, we will refer to these structural requirements, e.g., during our content analysis.

\section{$3 \quad$ Research design}

\subsection{Data collection}

Recalling our three research questions and in line with the explorative approach of our study, we decided to interview experts in the field of digital transformation of German enterprises to find answers to our research questions. Experts are individuals who provide domain-specific knowledge and skills obtained through multiple years of professional experience in a certain field. These individuals provide an adequate perspective on a subject matter, especially for explorative questioning [20].

In order to assure the validity of our data collection, experts from three different domains were selected (triangulation): mid- or top-level industry managers and executives from German enterprises dealing with digital transformation, senior consultants working on German digital transformation projects cross-industry and experienced researchers who investigate digital transformation in German companies academically. We thus followed a purposeful sampling. To be specific, in our case we conducted a maximum variation approach allowing for both exploring different angles on the topic and identifying important shared patterns that cut across cases [21]. The wide range of industrial sectors (ranging from automotive to IT and telecommunications), as well as the different company sizes (ranging from 1,000 to 400,000 employees), ensured that various challenges of digitisation were brought up in the interviews. In total, 16 semi-structured expert interviews were conducted.

All of the semi-structured interviews were carried out via Skype or over the phone. To ensure flexibility while guiding the interviews, we chose a set of open-ended questions (e.g., What are the structural requirements in the context of the digital transformation of a company?; What are the challenges on the way towards a digital enterprise?; What changes need to be made in a company to take advantage of the opportunities offered by digitisation?). Interview participation was voluntary, and anonymity was promised. The interviews were transcribed and took, on average, 40 minutes.

After eleven of the 16 interviews, a theoretical saturation was perceived. Such saturation indicates a redundancy of information [22]. That is, many of the respondents' answers were quite similar, even across the different domains of expertise (i.e., research, industry and consultancy). Such redundancy of information in the analysis suggests an adequate sample size [23]. This observation also underpins the reasoning of Guest et al. [24] that, in a relatively homogenous sample (i.e., in our case, similar in terms of expertise level), a theoretical saturation can be expected to occur after six to 12 interviews. 


\subsection{Data analysis}

For the analysis of the interview transcripts, we applied the 'Gioia methodology' [25]. This approach aims to address issues related to "qualitative rigor' and can be considered as an established approach for conducting qualitative research and is applied in various studies of high quality [e.g., 26].

The first analytic step according to Gioia et al. is building so-called first-order concepts. First-order concepts directly correspond with the interviewees' terminology; they should "adhere faithfully to informant terms" [25, p. 20]. Therefore, a passage of the text with a specific content was marked as a first-order concept. This was done for the entire corpus (i.e., 16 transcripts). For gaining a manageable number of first-order concepts, we searched for similarities and differences among the concepts and grouped them with suitable category labels. The QCAmap-Software ${ }^{2}$ supported us during this step in the coding and categorisation process and facilitated a subsequent transparent determination of intercoder-reliability. The initial coding procedure was conducted by two researchers independently. They agreed in $86 \%$ of the cases and clarified the remaining cases during a discussion with a third researcher.

The first-order concept set developed in the previous step was analysed regarding its structure and with the objective of developing more abstract second-order themes. Second-order themes 'help us describe and explain the phenomena we are observing' [25]. This step is challenging, as we as researchers must think at multiple levels simultaneously: at the interviewee's level and at the abstract second-order level. Because of this challenge, we carefully considered the DTF and its structural requirements (operational change, responsibility for digital transformation, organisational positioning of new activities, and building of organisational competencies) for developing second-order themes. This included two steps. First, we assigned fitting first-order constructs to one of the four extant structural requirements (e.g., the first-order concept 'new positions and roles' was assigned to the DTF requirement 'responsibility for digital transformation'). Second, we developed new second-order themes for those first-order concepts we were unable to assign to one of the existing requirements. At this stage, we considered the abstraction level of the four extant structural requirements and applied the same abstraction level when developing new requirements at the second-order level.

Finally, it was investigated whether second-order themes might be aggregated. This step is of minor importance, compared to first-order concept and second-order theme development, since the latter already aims to answer the research question [25]. However, we apply the aggregate dimension view for illustrating which themes are established (i.e., already part of the initial DTF) or novel.

\section{$4 \quad$ Findings}

The process diagram in Figure 1 depicts the outcomes of the various stages in the Gioia methodology. The illustration reveals all the structural requirements for digital

2 https://www.qcamap.org/ 
transformation, mentioned by the interviewees, following our data analysis approach. In total, 18 distinct requirements (i.e., first-order concepts) have been highlighted.

Thirteen of the 16 experts deemed agility, in terms of organisational structure, as significant for organisations in mastering the digital transformation. Change, in the process of organisational thinking, was expressed by eleven participants as a structural requirement for a digital transformation. Likewise, this code was mentioned most often, with a total of 22 instances in the 16 interviews. Nine of the 16 respondents identified a clear process structure as relevant in this context. The first-order concepts - i.e., IT infrastructure, continuous learning and new positions and roles - each came up in six of the expert interviews; the first-order concept digital HR was mentioned by seven experts. In each case, one-fourth of the respondents identified the following first-order concepts as requirements for organisations' digital transformation: focus on customer's needs, clear responsibilities, democratisation of decision making, a topdown process in decision implementation, flexibility, innovative learning methods, and data security.

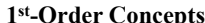

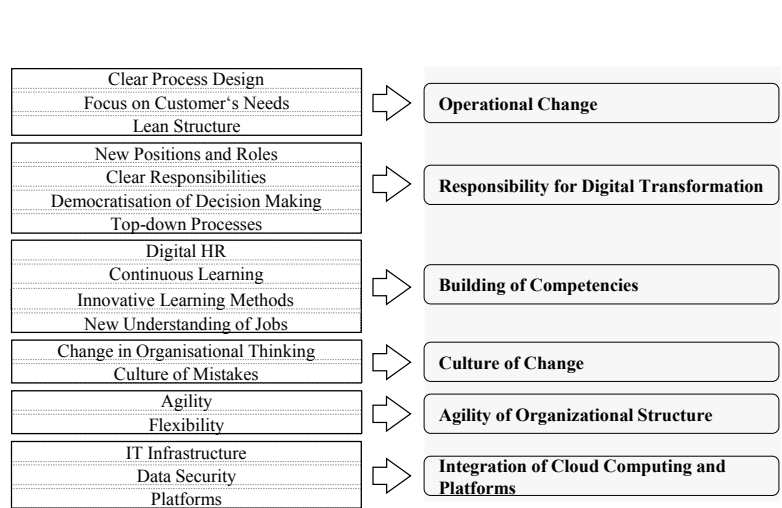

Aggregate Dimensions

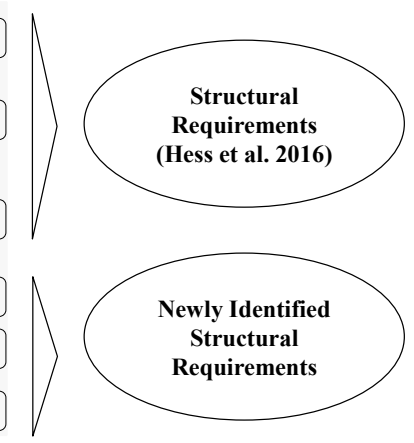

Fig. 1. Findings based on qualitative content analysis according to Gioia et al. (2013)

\section{Discussion}

\subsection{Comparison of our findings with the DTF}

In the DTF [2], four structural requirements are formulated. We were able to identify three of these four (i.e., operational change, responsibility for digital transformation, and building of organisational competencies), while one requirement was neither directly nor indirectly mentioned (i.e., organisational positioning of new activities). This is basically the answer on two of the three research questions, namely the question regarding those structural requirements identified in our cross-domain analysis which are already part in the DTF (RQ2), and the question regarding structural 
requirements from the initial DTF which could not be identified in the cross-domain analysis (RQ3).

Hess et al. [2] discuss three different sub-topics of operational change: products and services, business processes, and skills. We found all three aspects during our analysis. Products and services relate to statements, which we have coded as customer focus. Many respondents emphasised the meaning of clear and lean processes, which can be linked to business processes in the initial DTF.

The second key topic we found is responsibility for digital transformation. Similar to the subtopics suggested in DTF (i.e., group CEO, business unit CEO, group CDO, and group CIO), many respondents mentioned specific top management roles, particularly the Chief Digital Officer (CDO); thus supporting recent studies in which the rapid emergence of this new role has been investigated [7]. Furthermore, many respondents named aspects such as top-down decision making and a clear leadership strategy regarding digital transformation as important.

Also, the topic of building competencies could be identified in our analysis. Considering the suggested subtopics of the DTF (i.e., internally, partnerships, company takeovers, external sourcing), we were able to find statements mostly related to the building of competencies 'internally'. The interviewees often mentioned the topic of innovative learning approaches, e.g., using MOOCs or other online formats. They also emphasised that only a digital HR approach can facilitate building organisational competencies internally. Another aspect with high priority among the interviewees are the individual digital competencies (or skills) of employees, which is in line with current research on this topic [e.g., 8]. In this context, the interviewees mentioned continuous learning, with a focus on digital competencies (i.e., new understanding of jobs), as one of the key challenges in today's business world. Furthermore, freedom and trust were mentioned as important complementary aspects when developing competencies.

Although we were able to identify significant parts of the structural requirements mentioned in the DTF of Hess et al. (2016), one topic could not be found: organisational positioning of new activities (integrated vs. separated). This might be due to the different perspectives of the study by Hess et al. (2016) and our research. The authors of the initial DTF had a stronger focus on business model aspects and digital transformation as a whole. We, instead, place focus on the specific aspect of structural requirements for digital transformation.

\subsection{New structural requirements for digital transformation}

In addition to the topics already suggested in the DTF of Hess et al. [2], we were able to elaborate three new structural requirements for digital transformation: culture of change, agility of organisational structure and integration of cloud computing and platforms (see also Figure 2). This is the answer on RQ1, that asks for structural requirements identified in our cross-domain analysis which are not part of the initial DTF of Hess et al.

Eleven of the 16 interviewees mentioned a change of organisational culture as key for digital transformation. During the interviews, we asked how this rather abstract 
term might be made more concrete. We found that there seem to be two different understandings of a culture of change. The first meaning is similar to the recently popular notion of 'disruption'. But contrary to existing research on disruption [e.g., $27,28]$, the interviewees use the term simply to describe fundamental changes. In this context, a change culture indicates that an organisation, specifically its managers, allow or even seek proactive, basic alterations. One of the experts expresses this view in the following statement: 'It is important for an organisation [in order to profit from the opportunities of digital transformation] to engage in a radical rethinking and reorientation, to be able to change things and leave old structures to find new ways' (Interviewee \#7).

The second understanding of culture change that we could identify is a more evolutionary one. It is characterised by allowing mistakes and interpreting them as starting points for improvement, compared to a setting in which mistakes have a purely negative image. Besides this openness to experimentation and failing, no additional fundamental changes take place. A respondent formulates this idea as 'a culture and processes allowing that things go wrong, where you can experiment a lot, that is still positive, even if five projects fail' (Interviewee \#11).

The next new factor we were able to identify is the agility of organisational structure. Again, two different subtopics emerged. First, many respondents mentioned innovative organisational structures such as the liquid organisation. The interviewees emphasised the disappearance of classical functions and departments, replaced by a more project-oriented framework, in which employees are grouped according to their complementary competencies: 'They [organisations] should quit department structures and organise according to competencies" (Interviewee \#10). Freelancing replaces classical employee-employer relationships. Leaders focus on outcomes and interpret themselves as moderators instead of supervisors. This goes along with new approaches and tools such as Design Thinking and SCRUM.

Second, the other understanding of agility refers to a more stable and hierarchical organisational structure. While digital aspects are more and more integrated, organisations stick to their classical organisational chart; however, they are often accompanied by new roles. An expert stated that in order to be agile it is crucial to 'create new positions', such as 'a data scientist, community manager or digital consultant' (Interviewee \#14). In this context, these positions are not seen as being responsible for digital transformation, but of importance regarding organisational agility as they encourage quick responses to market opportunities.

Both experts' interpretations of agility of organisational structure rest on the firm's ability to adapt to new circumstances by realigning its structure and competencies. In line with recent definitions of agility in an organisational context [e.g., 29], the interviewees mention time-related aspects such as responding to new market opportunities quickly. Talking about agility, interviewees describe the structural requirements necessary for organisational agility such as specific approaches (e.g., Design Thinking and SCRUM) and related changes of the technological infrastructure, and new positions.

The last new structural requirement that we identified refers to the integration of cloud computing and platforms. The interviewees underlined the meaning of a 'sound 
and valuable' IT infrastructure (Interviewee \#4) and the need for cloud computing and 'platforms for exchanging information' (Interviewee \#7). Another very important aspect is data security: 'Data and IT security are obvious requirements for an organisation's digitalisation' (Interviewee \#7). Two sub-categories regarding cloud computing and platforms can be identified in terms of the ownership. Today, infrastructure, platforms, software, and even entire business processes can be obtained from external vendors. In this context, $[30,31]$ goes as far as to interpret IT as a commodity that does not provide any competitive advantage.

On the other hand, obtaining infrastructure, platforms, and services externally goes along with risks and challenges. For instance, customers might not want their private data handled by third-party companies. Thus, while the need for cloud computing and platforms is indisputable from the interviewees' perspective, a company should carefully balance the question of ownership.

Based on our analysis, we suggest the following enriched structural changes section of the DTF (see Figure 2). The shaded area indicates the new topics and respective strategic questions.

\begin{tabular}{|c|c|c|c|c|}
\hline \multicolumn{5}{|l|}{ Structural changes (enriched) } \\
\hline Responsibility for DT strategy? & Group CEO & Business Unit CEO & Group CDO & Group CIO \\
\hline $\begin{array}{l}\text { Organisational positioning of new } \\
\text { activities? }\end{array}$ & \multicolumn{2}{|c|}{ Integrated } & \multicolumn{2}{|c|}{ Separated } \\
\hline Operational changes? & Products and services & \multicolumn{2}{|c|}{ Business processes } & Skills \\
\hline Building of competencies? & Internally & Partnerships & Company takeovers & External sourcing \\
\hline Culture of change? & \multicolumn{2}{|c|}{ Disruptive } & \multicolumn{2}{|c|}{ Evolutionary } \\
\hline Agility of organisational structure? & \multicolumn{2}{|c|}{ Liquid organisation } & \multicolumn{2}{|c|}{ Hierarchical organisation } \\
\hline Cloud computing and platforms? & \multicolumn{2}{|c|}{ Ownership lies outside the firm } & \multicolumn{2}{|c|}{ Ownership lies with the firm } \\
\hline
\end{tabular}

Fig. 2. Enriched structural changes section of the DTF proposed by Hess et al. (2016)

\section{Conclusion}

While being one of the first empirical papers about the structural requirements for digital transformation of German companies, we have been able to explore new elements: culture of change, agility of organisational structure and integration of cloud computing and platforms. These requirements complement existing factors suggested by Hess et al. [2]. Thus, we can present a comprehensive set of structural requirements for digital transformation which is based on a cross-domain analysis, and we can develop an advanced version of the DTF, as shown in Figure 2. We discuss subtopics that were extracted from the interviews, which can be applied by managers to carefully analyse their organisations in terms of digital transformation.

Corresponding managerial implications of our study are manifold. Our findings indicate that digital transformation is a rather complex and multifaceted process. From a leadership perspective, a substantial understanding of various internal and external aspects is required. The extended DTF supports managers to cope with such demands, but it can only provide a starting point. Digital transformation, as implied in the term, is a dynamic phenomenon. Managers need to understand that there will be a continu- 
ous need for learning (e.g., regarding technological innovations). Thus, they must ensure a learning-friendly environment. Beyond that, managers must answer the questions of whether and how technological innovations might improve the effectiveness and efficiency of their organisation. Thus, a digital transformation project, as is often announced in companies, might be misleading. Instead, leaders should aim at implementing a dynamic and open-digital mindset in their companies.

The goal of this study was to explore structural requirements for the digital transformation of a company. The factors (i.e., requirements) we were able to identify might serve as the starting point for developing theory [4, 25]. However, this would require careful elaboration of causal relations between the constructs as well as their underlying dynamics [32]; this was not part of our study. Thus, further research efforts should be invested in an improved understanding of such factors, with a focus on their completeness, their relations and underlying mechanisms.

Considering the DTF of Hess et al. [2], we have focussed on one specific aspect (i.e., structural requirements) which is one out of four categories. While we think that the structural requirements are the most fundamental aspect of the DTF, a more comprehensive naturalistic evaluation would be to analyse the other categories of the DTF, as well.

Another limitation lies in the cultural context. We interviewed experts from Germany. Considering the distinct features of the German culture (e.g., regarding uncertainty avoidance or long-term orientation [33]), we believe that integrating data from other cultures might lead to different results. For instance, the category of culture of mistakes can be expected to be more common and accepted in countries with a lower uncertainty avoidance (e.g., USA) than in Germany [34, 35]. Also, the dimension of data security is likely to differ among countries. In Europe, for example, data security is widely established and in the focus of the public whereas, in the USA, it is much less emphasised. Considering the high score of Germany for the dimension of longterm orientation [34,35], it can be assumed that disruptive change is not favoured in this culture. The USA, again, is a suitable counterexample in terms of long-term orientation, as can be seen in the wide acceptance of disruptive behaviour in its culture [33]. Aside from cultural considerations on a country level, further research could focus on organisational culture [e.g., 36] which has also proved to be an important field of study in IS and management [37]. Therefore, future research in the field of digital transformation should focus on additional cultural contexts, as may serve as a basis for identifying and understanding such differences.

\section{References}

1. Legner, C., Eymann, T., Hess, T., Matt, C., Böhmann, T., Drews, P., Mädche, A., Urbach, N., Ahlemann, F.: Digitalization. Opportunity and Challenge for the Business and Information Systems Engineering Community. Business \& Information Systems Engineering 59, 301-308 (2017)

2. Hess, T., Matt, C., Benlian, A., Wiesböck, F.: Options for Formulating a Digital Transformation Strategy. MIS Quarterly Executive 15, 123-139 (2016) 
3. Veit, D., Clemons, E., Benlian, A., Buxmann, P., Hess, T., Kundisch, D., Leimeister, J.M., Loos, P., Spann, M.: Business Models. Business \& Information Systems Engineering 6, 45-53 (2014)

4. Hinings, B., Gegenhuber, T., Greenwood, R.: Digital Innovation and Transformation. An Institutional Perspective. Information and Organization 28, 52-61 (2018)

5. Windhagen, E., Bughin, J., Mischke, J., Baur, C., Mattern, F., Forman, S.: Stimulating digital adoption in Germany. Report McKinsey Global Institute (2017)

6. Matt, C., Hess, T., Benlian, A.: Digital Transformation Strategies. Business \& Information Systems Engineering 57, 339-343 (2015)

7. Horlacher, A., Hess, T.: What Does a Chief Digital Officer Do? Managerial Tasks and Roles of a New C-Level Position in the Context of Digital Transformation. In: Bui, T.X., Sprague, R.H. (eds.) Proceedings of the 49th Annual Hawaii International Conference on System Sciences. 5-8 January 2016, Kauai, Hawaii, pp. 5126-5135. IEEE, Piscataway, NJ, Piscataway, NJ (2016)

8. Murawski, M., Bick, M.: Digital competences of the workforce - a research topic? Business Process Management Journal 23, 721-734 (2017)

9. Fitzgerald, M., Kruschwitz, N., Bonnet, D., Welch: Embracing Digital Technology. A New Strategic Imperative. MIT Sloan Management Review, 1-12 (2013)

10. Kahre, C., Hoffmann, D., Ahlemann, F.: Beyond Business-IT Alignment - Digital Business Strategies as a Paradigmatic Shift: A Review and Research Agenda. Proceedings of the 50th Hawaii International Conference on System Sciences, 4706-4715 (2017)

11. Holotiuk, F., Beimborn, D.: Critical Success Factors of Digital Business Strategy. 13th Proceedings of International Conference on Wirtschaftsinformatik (WI 2017), 991-1005 (2017)

12. Hansen, R., Kien, S.S.: Hummel's digital transformation toward omnichannel retailing: key lessons learned. MIS Quarterly Executive 14, 51-66 (2015)

13. Chanias, S., Hess, T.: Understanding Digital Transformation Strategy Formation: Insights from Europe`s Automotive Industry. Pacific Asia Conference on Information Systems 2016 Proceedings, 296 (2016)

14. Chanias, S.: Mastering Digital Transformation: the Path of a Financial Services Provider Towards a Digital Transformation Strategy. Proceedings of the 25th European Conference on Information Systems (ECIS), Guimarães, Portugal (2017)

15. Peppard, J., Galliers, R.D., Thorogood, A.: Information systems strategy as practice: Micro strategy and strategizing for IS. The Journal of Strategic Information Systems 23, 1-10 (2014)

16. Henderson, J.C., Venkatraman, N.: Strategic alignment: Leveraging information technology for transforming organizations. IBM Systems Journal 32, 4-16 (1993)

17. Brynjolfsson, E., McAfee, A.: The Second Machine Age. Work, Progress, and Prosperity in a Time of Brilliant Technologies. W.W. Norton \& Company, New York, London (2014)

18. McAfee, A., Brynjolfsson, E.: Machine, platform, crowd. Harnessing our digital future. W.W. Norton \& Company, New York (2017) 
19. Bharadwaj, A., El Sawy, O., Pavlou, P.A., Venkatraman, N.: Digital Business Strategy: Toward a Next Generation of Insights. MIS Quarterly 37, 471-482 (2013)

20. Bernard, H.R.: Social research method. Qualitative and quantitative approaches. SAGE Publications, Thousand Oaks, Calif. (2013)

21. Palinkas, L.A., Horwitz, S.M., Green, C.A., Wisdom, J.P., Duan, N., Hoagwood, K.: Purposeful Sampling for Qualitative Data Collection and Analysis in Mixed Method Implementation Research. Administration and policy in mental health 42 , 533-544 (2015)

22. Hennink, M., Hutter, I., Bailey, A.: Qualitative Research Methods. SAGE Publications Ltd, London (2011)

23. Mason, J.: Qualitative Researching. Sage, Los Angeles (2012)

24. Guest, G., Bunce, A., Johnson, L.: How Many Interviews Are Enough? Field Methods 18, 59-82 (2006)

25. Gioia, D.A., Corley, K.G., Hamilton, A.L.: Seeking Qualitative Rigor in Inductive Research. Organizational Research Methods 16, 15-31 (2013)

26. Smith, W.K.: Dynamic Decision Making. A Model of Senior Leaders Managing Strategic Paradoxes. Academy of Management Journal 57, 1592-1623 (2014)

27. Christensen, C.M.: Innovator's Dilemma: When New Technologies Cause Great Firms to Fail (management of innovation and change series). Harvard Business Review (1997)

28. Denning, S.: Christensen updates disruption theory. Strategy \& Leadership 44, 10-16 (2016)

29. Ravichandran, T.: Exploring the Relationships Between IT Competence, Innovation Capacity and Organizational Agility. The Journal of Strategic Information Systems 27, 22-42 (2018)

30. Carr, N.G.: IT Doesn't Matter. Harvard Business Review 81, 41-49 (2003)

31. Carr, N.G.: The Big switch. Rewiring the world, from Edison to Google. W. W. Norton, New York, London (2009)

32. Whetten, D.A.: What Constitutes a Theoretical Contribution? The Academy of Management Review 14, 490-495 (1989)

33. House, R.J., Dorfman, P.W., Javidan, M.: Strategic leadership across cultures. The GLOBE study of CEO leadership behavior and effectiveness in 24 countries. SAGE Publications, Los Angeles (Calif.) (2013)

34. Hofstede, G.: What about Germany?, https://geert-hofstede.com/germany.html

35. Hofstede, G., Hofstede, G.J., Minkov, M.: Cultures and organizations. Software for the mind. McGraw-Hill (2010)

36. Schein, E.H.: Organizational Culture and Leadership. Jossey-Bass, San Francisco (2010)

37. Kummer, T.-F., Leimeister, J.M., Bick, M.: On the Importance of National Culture for the Design of Information Systems. Business \& Information Systems Engineering 4, 317-330 (2012) 